\title{
Behind the curve: Generating and analysing nets and tessellations on periodic minimal surfaces in their universal covering space
}

\author{
M. C. Pedersen ${ }^{1}$, J. J. K. Kirkensgaard ${ }^{1,2}$, S. T. Hyde ${ }^{3}$ \\ ${ }^{I}$ Niels Bohr Institute, University of Copenhagen, ${ }^{2}$ Department of Food Science, University of Copenhagen, ${ }^{3}$ School of Chemistry, \\ University of Sydney \\ mcpe@nbi.ku.dk
}

The in-surface crystallography of the family of three-periodic minimal surfaces (TPMS) consisting of the Primitive, Diamond, and Gyroid surfaces has been explored in detail in recent decades [1,2]. We begin by reviewing the underlying group theory and geometry as well as the relationship between the TPMS and their universal covering space, hyperbolic two-space, the fundamentals of which are shown in Figure 1(a).

We describe how these methods can be used to tailor the topology and geometry of three-periodic nets realised as embeddings commensurate with the symmetries of these surfaces [3-5] as shown in Figure 1(b). Using these ideas, we demonstrate how a number of nets with pre-specified topological properties are readily produced in this manner and assess their relevance for further study in the context of reticular chemistry and soft matter materials science.

Finally, we present preliminary results on visualisation methods for understanding how these patterns and nets are realised in simulations of liquid crystals in bulk [6] and confined polymer systems. Using an array of methods from computational geometry, we visualise these simulations in hyperbolic two-space to facilitate easy comparisons and further analysis as shown in Figure 1(c).

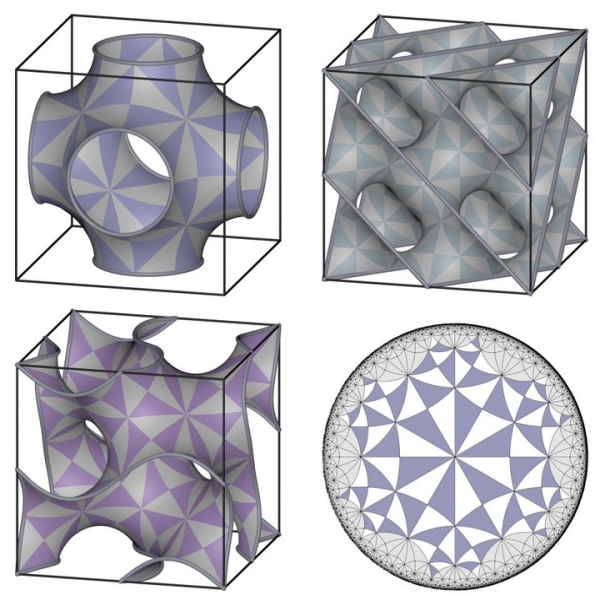

(a)

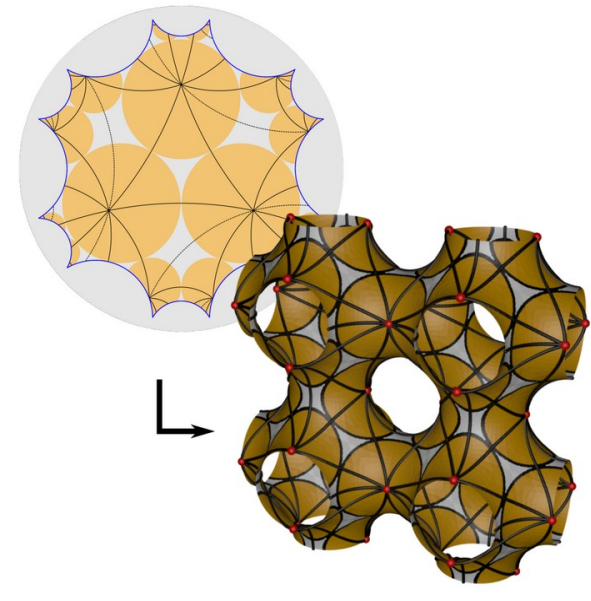

(b)

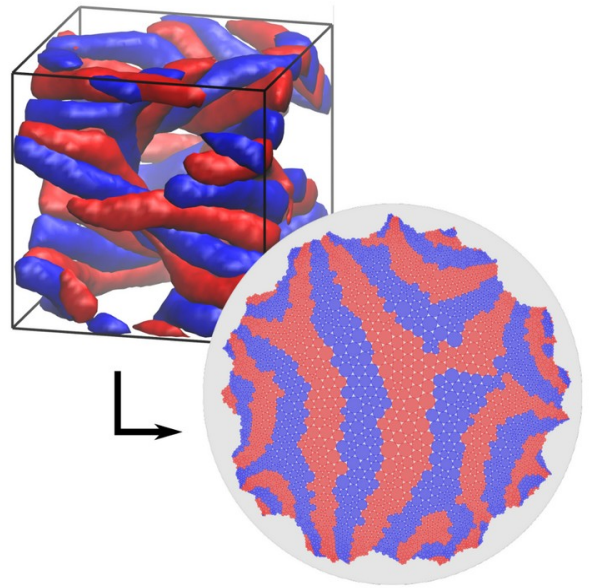

(c)

Figure 1. In (a), three renderings of the Primitive, Diamond, and Gyroid three-periodic minimal surfaces in their conventional unit cell. Also shown is a hyperbolic dodecagon (visualized using the Poincare disk model of hyperbolic two-space) corresponding to the primitive unit cell of these surfaces. In (b), a ten-connected net with symmetries commensurate with the surfaces in (a) is "lifted" to a three-periodic net in euclidean three-space by embedding the net on a TPMS. In (c), a structure emerging in a simulation of a bulk polymer system yielding a Gyroid-like film for the minority polymer components and a representation of this pattern in hyperbolic two-space by way of numerical (quasi-)conformal mapping.

[1] Sadoc, J.-F. \& Charvolin, J. (1989). Acta Crystallogr. A 45, 10-20.

[2] Robins, V., Ramsden, S., \& Hyde, S. T. (2004). Eur. Phys. J. B 39(3), 365-375.

[3] Pedersen, M. C. \& Hyde, S. T. (2018). Proc. Natl. Acad. Sci. U. S. A. 115(27), 6905-6910.

[4] Pedersen, M. C., Delgado-Friedrichs, O., \& Hyde, S. T. (2018). Acta Crystallogr. A 74(3), 223-232.

[5] Hyde, S. T. \& Pedersen, M. C. (2021). Proc. Roy. Soc. A 477, 20200372.

[6] Kirkensgaard, J. J. K., Evans, M. E., de Campo, L., \& Hyde, S. T. (2014). Proc. Natl. Acad. Sci. U. S. A. 111(4), 1271-1276.

Keywords: hyperbolic geometry; symmetry groups; chemical nets; graph embeddings; minimal surfaces

The authors thank Anders Dahl, Myfanwy Evans, Olaf-Delgado Friedrichs, Benedikt Kolbe, Stuart Ramsden, Vanessa Robins, Gerd Schröder-Turk, and Monique Teillaud for discussions and feedback on these topics and results. 